\title{
The Structural Properties of Even Free Graphs
}

\author{
T.Chalapathi \\ Assistant Professor Dept. of Mathematics Sree \\ Vidyanikethan Eng.College Tirupati,-517502 \\ Andhra Pradesh, India
}

\author{
R.V.M.S.S.Kiran Kumar \\ Research Scholar Dept. of Mathematics \\ S.V.University Tirupati,-517502 Andhra Pradesh, \\ India
}

\begin{abstract}
In this paper we introduced Group theoretic graph $\mathcal{E}_{2 n}$, even free graph associated with the finite abelian $\operatorname{group}\left(Z_{2 n}, \oplus\right)$ for each $n \geq 1$, also we explore structural properties of $\mathcal{E}_{2 n}$ and including enumeration of edges and cycles in $\varepsilon_{2 n}$.
\end{abstract}

\section{General Terms}

Graph theory, Group theory and Combinatorics.

\section{Keywords}

Even integers group, Even free graph, Cycles, Bipartite and Complete Bipartite graph.

\section{INTRODUCTION}

For standard terminology and notation in graph theory we refer Vitaly [10] and Harary [6]. We look up Bhattacharya [2] for group theoretic properties. A graph $G$ is a pair $(V, E)$,where $V$ is a non-empty finite set, and $E$ is a set of unordered pairs $(x, y)$ of elements $x$ and $y$ of $V$.The elements of $V$ are called the vertices of $G$ and the elements of $E$ are the edges of $G$.The study of algebraic structures using the properties of graphs has become an explicating research topic in the last twenty years .There are many papers on assigning a graph to a group, see [2,7]. The text books on algebraic graph theory by Godsil and Royal [5] provide much information about graphs and groups. The structure and various structural properties of algebraic graphs have been studied in literature see $[9,12]$

In this paper, we consider even free graph of the group of additive integers modulo $2 n$. This graph is inter related between two special branches of Mathematics, namely Group theory and Combinatorics.

\section{NOTATION AND BASIC PROPERTIES}

In this section we set up the notation, include some useful definitions and observe a few basic properties that follow directly from the definitions.

In this text all groups are assumed to be non-zero, finite commutative and with 1.As usual all graphs are finite simple undirected graphs.

For each positive integer $n \geq 1$, the algebraic structure

$\left(Z_{2 n}, \oplus\right)$ is a finite abelian group with respect to integers modulo $2 n$. Further $S=\left\{2 x: x \in Z_{n}\right\}$ is a subgroup of $\left(Z_{n}\right.$, $\bigoplus$ ), which is called even integers group of modulon Especially we have $S=Z_{2 n-1}$ but $S \neq Z_{n}$ for $n \geq 1$.

The degree of a vertex $u, \operatorname{deg}(u)$ in $G$ is the number of edges incident at $u$.If degree of each vertex is equal, say $r$ in $G$,then $G$ is called $r$-regular graph. Vertices $u$ and $v$ of a graph $G$ are adjacent if $(u, v) \in E(G)$.

A walk in a graph $G$ is an attending sequence of vertices and edges beginning and ending with vertices. A closed walk in which all the vertices are distinct is called a cycle. It is written as

$$
C=\left(v_{1}, v_{2}, v_{3} \ldots v_{n}, v_{1}\right) .
$$

A graph is called connected if any two vertices are connected by some path and a graph in which very pair of vertices is an edge is called complete. A graph $G$ is called bipartite if its vertex set $V$ can be partitioned into two disjoint sets $V_{1}$ and $V_{2}$ called parts in such a way that every edge connects vertices from different sets. A complete bipartite graph is a bipartite graph in which every vertex from part $V_{1}$ is adjacent to every vertex from part $V_{2}$.

\section{THE STRUCTURE AND PROPERTIES OF THE EVEN FREE GRAPH}

In this section we introduce the notation of the even free graph associated with $\operatorname{group}\left(Z_{2 n}, \oplus\right)$. For each $n \geq 1$ there exist several even free graphs .These graphs are motivated from Mangoldt graphs [8].Now we study the structure and several properties of these even free graphs.

\subsection{Definition}

For each positive integer $n \geq 1$ the even free graph $\varepsilon_{2 n}=$ $G(V, E)$ is the graph whose vertex set is $V=Z_{2 n}$ and in which edge set $E$ is the set of unordered pairs $(x, y)$ is an edge if and only if $x$ and $y$ are distinct elements of $Z_{2 n}$ such that $x+y$ is free from an even integer of modulo $2 n$.

The graphs $\mathcal{E}_{2 n-1}$ and $\mathcal{E}_{2 n+1}$ are both null graphs for $\operatorname{each} n \geq 1$. For this reason, the graph $\varepsilon_{n}$ is connected if and only if $n$ is even. Throughout the text we consider the graph $\mathcal{E}_{2 n}$ for each positive integer $n \geq 1$.

Two examples of even free graphs $\mathcal{E}_{4}$ and $\mathcal{E}_{6}$ are displayed in following figures respectively. 

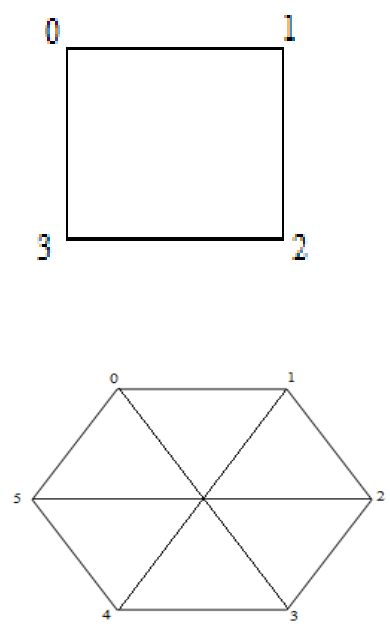

\subsection{Proposition}

Let $v$ be any vertex of the even free graph $\mathcal{E}_{2 n}$, then its degree, $\operatorname{deg}(v)=n$.

Proof: Let $u, v \in Z_{2 n}$ then $(u, v)$ is an edge of the graph $\varepsilon_{2 n}$ if and only if $u+v$ is free from an even integer. So the vertex $v$ can be written as $v=2 n-1-u$ for any $n \geq 1$. Thus the vertex $v$ is adjacent to all the distinct vertices of the type $2 n-1-u$ in $\varepsilon_{2 n}$. If $u$ is either odd or even, then the number of vertices of the type $2 n-1-u$ in $\mathcal{E}_{2 n}$ is equal to

$$
\frac{\left|Z_{2 n}\right|}{2}=n \text {. }
$$

Thus the number of edges incident at the vertex $v$ is $n$, and hence $\operatorname{deg}(v)=n$.

This proposition has a number of useful consequences.

\subsection{Corollary}

The even free Graph $\mathcal{E}_{2 n}$ is $n$-regular for all $n \geq 1$.

Proof: The degree of a vertex $v, \operatorname{deg}(v)$ in $\varepsilon_{2 n}$ is the number of edges incident at $v$.Due to Proposition 2.2, the degree of each vertex is equal and it is $n$. Hence $\mathcal{E}_{2 n}$ is $n$ - regular for each $n \geq 1$.

Next we establish enumeration of edges in the even free graph $\mathcal{E}_{2 n}$.

\subsection{Corollary}

The total number of edges in the even free graph $\varepsilon_{2 n}$ is $n^{2}$.

Proof: The total number of edges of $\varepsilon_{2 n}$ is half the total degree of $\varepsilon_{2 n}$. From Proposition 2.2, all vertices of $\varepsilon_{2 n}$ have the same degree; we simply multiply the degree of a vertex of $\varepsilon_{2 n}$ by $\frac{n}{2}$. Thus the total number of edges in $\varepsilon_{2 n}$ is equal to

$$
\frac{n}{2}\left|Z_{2 n}\right|=\frac{n}{2}(2 n)=n^{2} .
$$

\subsection{Corollary}

The graph $\mathcal{E}_{2 n}$ is Eulerian for each even integer $n$.
Proof: The graph is Eulerian if and only if every vertex of graphs is of even degree [6].From Proposition 2.2, $\operatorname{deg}(v)=$ $n$ for each vertex of $\varepsilon_{2 n}$.If $n$ is even, and then $\operatorname{deg}(v)$ is even. Hence the graph $\varepsilon_{2 n}$ is Eulerian if and only if $n$ is even. Further $\varepsilon_{2 n}$ is non - Eulerian if and only if $n$ is odd.

\subsection{Example}

The graphs $\varepsilon_{12}$ and $\varepsilon_{14}$ are Eulerian and non - Eulerian graphs respectively.

\section{ENUMERATION OF CYCLES}

In this section we enumerate number of odd and even cycles, and to study the complete bipartite property of the graph $\varepsilon_{2 n}$.

A triad $(x, y, z)$ is a triangle if and only if the pairs $(x, y),(y, z),(z, x)$ are edges of the undirected graph, and the triangle is also called 3-cycle and its length is 3 , and hence it is the shortest cycle of any undirected graph.

The following theorem illustrates the enumeration of triangles, and hence to enumerate the total number of odd cycles in the even free graph $\varepsilon_{2 n}$ for each $n \geq 1$.

\subsection{Theorem}

The number of triangles in the even free graph $\varepsilon_{2 n}$ is zero.

Proof: Let $u, v, w \in Z_{2 n}$. Then $u, v$ and $w$ are any three vertices of the graph $\varepsilon_{2 n}$. For any $u, v, w \in Z_{2 n}$, we have atleast one of $u+v, v+w, w+u$ is an even integer, it follows that atleast one of the pair $(u, v),(v, w),(w, u)$ is not an edge of the graph $\varepsilon_{2 n}$. Thus a triad $(u, v, w)$ is not a triangle in the graph $\varepsilon_{2 n}$.Hence the total number of triangles in the even free graph $\varepsilon_{2 n}$ is zero.

An immediate consequence of theorem worth nothing is what occurs when $k$ - cycle exists in $\varepsilon_{2 n}$ where $k$ is odd.We see that if $k$ is odd, then $\varepsilon_{2 n}$ does not contain an odd cycle. This gives $\mathcal{E}_{2 n}$ is a bipartite graph. There is a special relation between bipartite graphs and cycles, and it states that a graph is bipartite if and only if it has no odd cycles [6] .Hence $\mathcal{E}_{2 n}$ is bipartite graph.

\subsection{Theorem}

The even free graph $\mathcal{E}_{2 n}$ is complete bipartite graph for each $n \geq 1$.

Proof: For each $n \geq 1$, the set $V=Z_{2 n}$ is the vertex set of the graph $\mathcal{E}_{2 n}$. It can be partitioned into two non-empty disjoint subsets $V_{1}$ and $V_{2}$ such that

$$
\begin{aligned}
& V_{1}=\{0,2,4, \ldots, 2 n-2\} \text { and } \\
& V_{2}=\{1,3,5, \ldots, 2 n-1\}
\end{aligned}
$$

Here every edge of $\varepsilon_{2 n}$ joining a vertex of $V_{1}$ and a vertex of $V_{2}$. Hence $\varepsilon_{2 n}$ is complete bipartite graph.

\subsection{Example}

The even free graph and its complete bipartite graph of $\mathcal{E}_{8}$ are given below. 

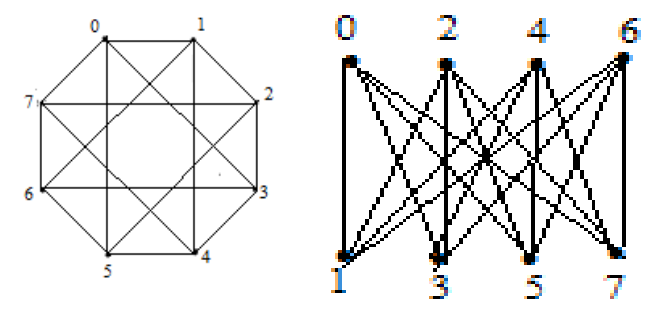

The following theorem illustrates the enumeration of $k-$ cycles in the even free graph $\mathcal{E}_{2 n}$ for each $n \geq 2$, where $k$ is even.

\subsection{Theorem}

The total number of $k$ - cycles in the graph $\varepsilon_{2 n}$ is $\left(n_{C_{k / 2}}\right)^{2}$ where $k$ is even and $4 \leq k<2 n$.

Proof: Let $n \geq 2$ be a positive integer and $k \geq 4$ be an even positive integer then the $k$-cycles in the graph $\varepsilon_{2 n}$ is either of the form $(0,1,2,3, \ldots k, 0)$ or $(0, k, k-1, \ldots, 1,0)$. Either of these two cycles shows that between any two odd integers there exist an even integer and vice versa. Since $k \geq 4$ is an even integer .It follows that $\frac{k}{2}$ is either even or odd integer .So that the $k$ - cycle in the graph $\varepsilon_{2 n}$ contains $\frac{k}{2}$ even and $\frac{k}{2}$ odd integers. The number of arrangements of $\frac{k}{2}$ odd integers are arranged between different fixed $\frac{k}{2}$ even integers from a collection of $n\left(=\frac{2 n}{2}\right)$ integers is $n_{C_{k / 2}}$ and similarly each one of these arrangements is a $k$ - cycle of the graph $\varepsilon_{2 n}$. So by the product rule of Combinatorics $[\mathbf{1}, \mathbf{1 1}$, the total number of $k$ cycles in the graph $\varepsilon_{2 n}$ is

$$
\left(n_{C_{k / 2}}\right)\left(n_{C_{k / 2}}\right)=\left(n_{C_{k / 2}}\right)^{2}
$$

\subsection{Example}

Forn $=3$, the total number of 4-cycles in the graph $\varepsilon_{6}$ is 9 , which are represented the following vertex sequences.

$$
\begin{aligned}
& (0,1,2,3,0),(0,1,2,5,0),(0,3,2,5,0) \\
& (0,1,4,3,0),(0,1,4,5,0),(0,3,4,5,0) \\
& (1,2,3,4,1),(1,2,5,4,1),(2,3,4,5,2) .
\end{aligned}
$$

\section{CONCLUSION}

In this paper, we have introduced a new graph structure, called even free graph. An important outcome of this paper is that for all even values to $2 n$ of $n \geq 1$, even free graph is regular simple undirected graph. Some other properties related to degree of vertices, enumeration of edges and cycles, and complete bipartite property has also been examined. It will be interesting to find out the number of Hamilton cycles in the even free graph.

\section{ACKNOWLEDGMENTS}

The authors express their sincere thanks to Prof.L.Nagamuni Reddy and Prof.S.Vijaya Kumar Varma for his suggestions during the preparation of this paper and the referee for his suggestions.

\section{REFERENCES}

[1] Alan Tucker., - Applied Combinatorics, John Wiley and Sons Pet Ltd, Singapore.

[2] Bhattacharya, P.B., Jain, S.K., and Nagpaul, S.N.,- Basic Abstract Algebra, Cambridge University Press, 2009, India.

[3] Chalapathi, T., Madhavi. and Venkataramana,S., Enumeration of Triangles in a Divisor Cayley Graphs, MEJS, 2013, 163-173.

[4] Chalapathi,T., - Studies on Enumeration Methods of Triangles and Hamilton Cycles in Cayley Graphs, Doctoral Thesis submitted to S.V.University, Tirupati, (2013), India.

[5] Godsil, C., and Royle,G., - Algebraic Graph Theory, Graduate Texts in Mathematics, 2001, Springer.

[6] Harary, F., - Graph Theory, Addition-Wesley Publ. Comp., 1969, Regarding, Massachusetts.

[7] Judson, T.W., - Abstract Algebra, PWS Publishing Company, 1994, Boston.

[8] Madhavi., and Chalapathi, T., - Enumeration of Basic Hamilton Cycles in the Mangoldt Graph, IJCA (2014), 99, 44-48.

[9] Norman L. Biggs., - Discrete Mathematics, Oxford University Press, 2003, India.

[10] Vitaly I. Voloshin. - Introduction to Graph Theory, Nova Science Publishers, 2009, Inc. New York.

[11] Van Lint, J.H., and Wison, R.M., - A Course in Combinatorics, Cambridge University Press, 2007, India.

[12] West, D.B., - Introduction to Graph Theory, Prentice Hall of India. 